\title{
Accurate control of a liquid-crystal display to produce a homogenized Fourier transform for holographic memories
}

\author{
Andrés Márquez, ${ }^{1, *}$ Sergi Gallego, ${ }^{1}$ David Méndez, ${ }^{1}$ Mariela L. Álvarez, ${ }^{1}$ Elena Fernández, ${ }^{2}$ \\ Manuel Ortuño, ${ }^{1}$ Cristian Neipp, ${ }^{1}$ Augusto Beléndez, ${ }^{1}$ and Inmaculada Pascual ${ }^{2}$ \\ ${ }^{1}$ Departamento de Física, Ingenierýa de Sistemas y Teoría de la Señal, Universidad de Alicante, Apartado 99, 03080 \\ Alicante, Spain \\ ${ }^{2}$ Departamento Interuniversitario de Óptica, Universidad de Alicante, Apartado 99, 03080 Alicante, Spain \\ *Corresponding author: Andres.Marquez@ua.es
}

Received June 5, 2007; accepted July 11, 2007;

posted July 24, 2007 (Doc. ID 83711); published August 17, 2007

\begin{abstract}
We show an accurate procedure to obtain a Fourier transform (FT) with no dc term using a commercial twisted-nematic liquid-crystal display. We focus on the application to holographic storage of binary data pages, where a drastic decrease of the dc term in the FT is highly desirable. Two different codification schemes are considered: binary $\pi$ radians phase modulation and hybrid ternary modulation. Any deviation in the values of the amplitude and phase shift generates the appearance of a strong dc term. Experimental results confirm that the calculated configurations provide a FT with no dc term, thus showing the effectiveness of the proposal. (C) 2007 Optical Society of America

OCIS codes: $050.1970,070.2590,210.2860,230.3720,230.6120$.
\end{abstract}

Liquid-crystal displays (LCDs) [1] are interesting devices to be used as spatial light modulators (SLMs) in holographic data storage systems (HDSS) [2], where they enable the real-time update of the data page to be recorded. The data page is usually displayed as a binary amplitude transparency and its Fourier transform (FT) is holographically recorded in a photosensitive material. A highly inhomogeneous FT is obtained with a very energetic zero frequency (or dc term), which may saturate the dynamic range of the material, thus producing a decrease in the signal-tonoise ratio in the reconstruction and limiting the accessible dynamic range [3]. Various alternative schemes have been proposed, such as recording the defocused FT. However, this limits the performance of a holographic memory in one of its most powerful applications, i.e., as associative memories, and lowers the storage density. Another alternative proposed is to use random or pseudorandom phase masks to uniformize the FT spectrum [3]. In this case, the main drawback is the stringent requirement for optical alignment between the phase mask and the LCD with subpixel accuracy. The problems given by the lack of homogeneity of the FT can be solved in part by using some other modulation schemes, such as hybrid ternary modulation (HTM) $[4,5]$ or binary $\pi$ radians phase-only modulation $(\pi \mathrm{BPM})[6,7]$.

In general, commercially available LCDs are based on the twisted-nematic (TN) configuration [1]. In TN-LCDs, the amplitude and the phase modulations are coupled. Present LCDs have a larger resolution, forcing a decrease in the thickness of the device to provide a faster response for addressing the pixels. The decrease in the thickness shortens the dynamic modulation range of the device. An optimization procedure leading to amplitude-only or phase-only modulations with thin LCDs was demonstrated in [8]. This optimization procedure is based on an ap- proximate model describing the physical properties of the LCD [9], and on the use of elliptically polarized light and short wavelengths. The high accuracy of the calculations made with this model enable an accurate control of the amplitude and phase of the wavefront.

In this work we show a method to obtain optimum $\pi \mathrm{BPM}$ or HTM configurations using a LCD. In our strategy the accuracy demonstrated by the model we use to describe the modulation properties of the LCD $[8,9]$ is essential, because it enables a reliable computer search for the optimum modulation configurations. Some final adjustments in the voltage levels may be necessary, which can be easily performed using binary phase gratings written onto the LCD. We confirm experimentally that the calculated configurations produce the FT of a binary data page with a very good homogeneity.

In the experiments we use a Sony LCD model LCX012BL, extracted from a video projector Sony VPL-V500. We use the electronics of the video projector to send the voltage to the pixels of the LCD. The LCD is illuminated with the spatially filtered, expanded, and collimated beam from a laser Nd-YVO with a wavelength of $532 \mathrm{~nm}$. The setup for generation and detection of elliptically polarized light considers two sets composed of a linear polarizer and a wave plate retarder, one set in front and one set behind the LCD [8]. The angles of the polarizer and the wave plate in front of the LCD, $\varphi_{1}$ and $\eta_{1}$, respectively, are referred to the orientation of the director at the input surface, whereas the angles of the elements behind the LCD, $\varphi_{2}$ and $\eta_{2}$, are measured with respect to the orientation of the director at the output surface. The measured retardances for the wave plates at $532 \mathrm{~nm}$ are $88^{\circ}$ and $90^{\circ}$, respectively, for the input and the output wave plate, with their fast axis aligned with the zero of the rotary stage. 
The model in [9] provides an analytic expression for the Jones matrix describing the performance of the LCD. Once the values for the parameters of the LCD have been calibrated [9], we apply a computer search to calculate through an optimization procedure the orientation of the polarizing devices in front of and behind the LCD to obtain specific modulation regimes. In the optimization process the magnitudes $\eta_{1}, \eta_{2}, \varphi_{1}$, and $\varphi_{2}$ are left as free parameters. The figure of merit to be optimized depends on the modulation regime in which we have interest.

First, we look for a binary phase-only modulation regime with a high efficiency and that is able to provide two phase levels with a difference of $180^{\circ}$, which represent the two bit levels in the data page in $\pi \mathrm{BPM}$. The values obtained for the angles of the polarizing elements are: $\varphi_{1}=-37^{\circ}, \eta_{1}=0^{\circ}, \eta_{2}=0^{\circ}$, and $\varphi_{2}=38^{\circ}$. In Fig. 1(a) we show the theoretically predicted normalized intensity and phase shift as a function of the gray level. We observe that the transmission is almost flat and maximum, and the phase-shift modulation depth is close to $270^{\circ}$. Using gray levels 0 and 190 we obtain a $\pi \mathrm{BPM}$ configuration.

For HTM the LCD is required to show two gray levels with a similar transmission and with a $180^{\circ}$ phase difference (representing the white level in the data page) and a low transmission level (representing the black level). The values obtained for the angles of the polarizing elements are: $\varphi_{1}=153^{\circ}, \eta_{1}$ $=126^{\circ}, \eta_{2}=127^{\circ}$, and $\varphi_{2}=0^{\circ}$. In Fig. $1(\mathrm{~b})$ we show the theoretically predicted normalized intensity and phase shift. The black level is at gray 250 and the two white levels are at gray 0 and 190, respectively. Theoretically, the phase difference between the white
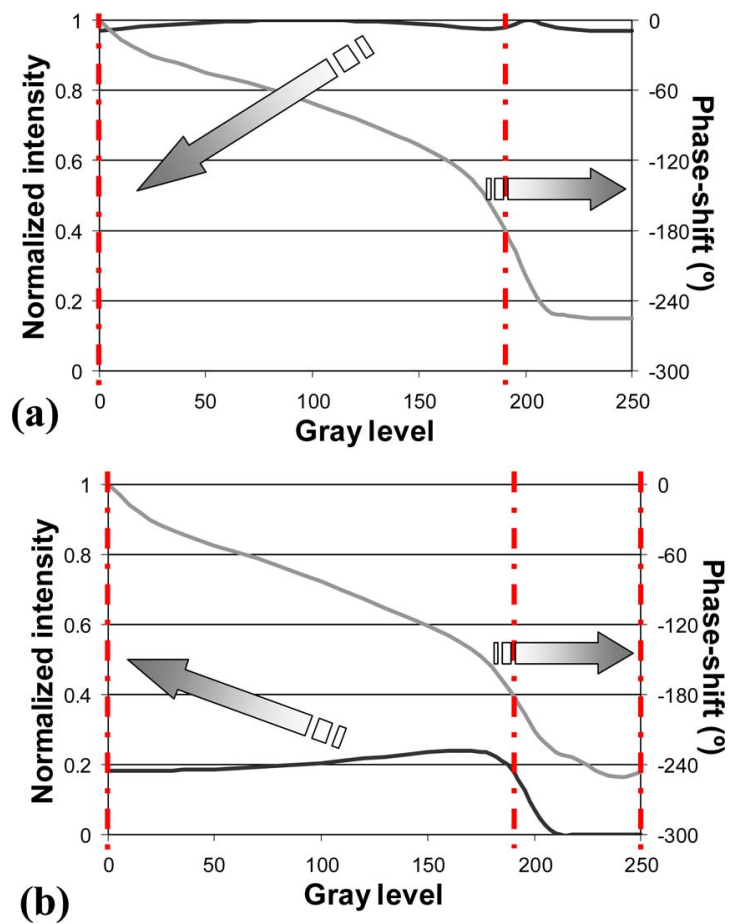

Fig. 1. (Color online) Normalized intensity and phaseshift modulation. (a) $\pi \mathrm{BPM}$ configuration; (b) HTM configuration. Vertical lines indicate the gray levels selected to realize each of the modulation regimes. levels is $180^{\circ}$ and the intensity difference normalized by the average intensity of the two levels is lower than $1 \%$. The experimental intensity contrast between the white levels and the black level is close to 1:100. The HTM imposes more severe restrictions on the optimization process than the $\pi \mathrm{BPM}$. As a result, the normalized intensity transmission obtained for the white levels in HTM is not very high (0.18). A thicker LCD and/or the use of a shorter wavelength would increase the dynamic modulation range of the LCD. As a consequence, a larger set of states would become available in the optimization process and a higher intensity transmission for the white levels would be available [5].

Some slight adjustments must be done on the gray levels selected from the modulation curves in Fig. 1. Small deviations of the values for the amplitude and/or phase shift produce the appearance of an important dc term in the FT. To minimize any deviation in the phase-shift values we follow a simple approach. We generate binary diffraction gratings whose two gray levels correspond to the two levels with a $\pi$ radians phase difference given in Fig. 1. In both modulation regimes considered in this work these two gray levels are 0 and 190 . When addressed onto the LCD the zero diffracted order disappears if the phase difference between the two levels is exactly $\pi$ radians. Experimentally we measure a very low zero order; however, it can still be lower when slightly shifting the upper gray level. Eventually, we have obtained that gray levels 0 and 198 for $\pi \mathrm{BPM}$, and 0 and 193 for HTM provide a better extinction of the zero order.

In this experiment with the binary gratings a stop is placed before the LCD limiting the aperture of the incident collimated beam to $\sim 20 \mathrm{~mm}$ (480 LCD pixels), and we place a CCD camera, Hamamatsu C5403, at the focal plane of a $20 \mathrm{~cm}$ doublet lens. The captured image is digitized by a frame grabber, Matrox Meteor, connected to a personal computer. We note that this setup is also used in the experiment to obtain the images in Fig. 2. When realizing the experiences with the binary gratings, the +1 and -1 orders are highly saturated to gain a major sensitivity in the extinction of the zero order. We addressed both horizontal and vertical diffraction gratings and with different periods $(16,8,4$, and 2 pixels/period) to verify if the LCD unit used in this work exhibits the anamorphic frequency dependent effect reported in [10]. For the vertical gratings, independently of the frequency, we obtain a good extinction of the zero order applying the same gray-level values. For the horizontal gratings some slight adjustments have to be made in the gray levels as the frequency of the grating increases. Thus, a slight anamorphic frequency dependent effect is exhibited by the LCD used in this work. For the horizontal gratings with a 2 pixels/ period there was no gray-level values combination able to extinguish the zero order.

To experimentally analyze the FT of binary data pages to be stored in a HDSS we generate random binary images with an equal number of 0's and 1's, using the optimum gray levels previously calculated: 0 

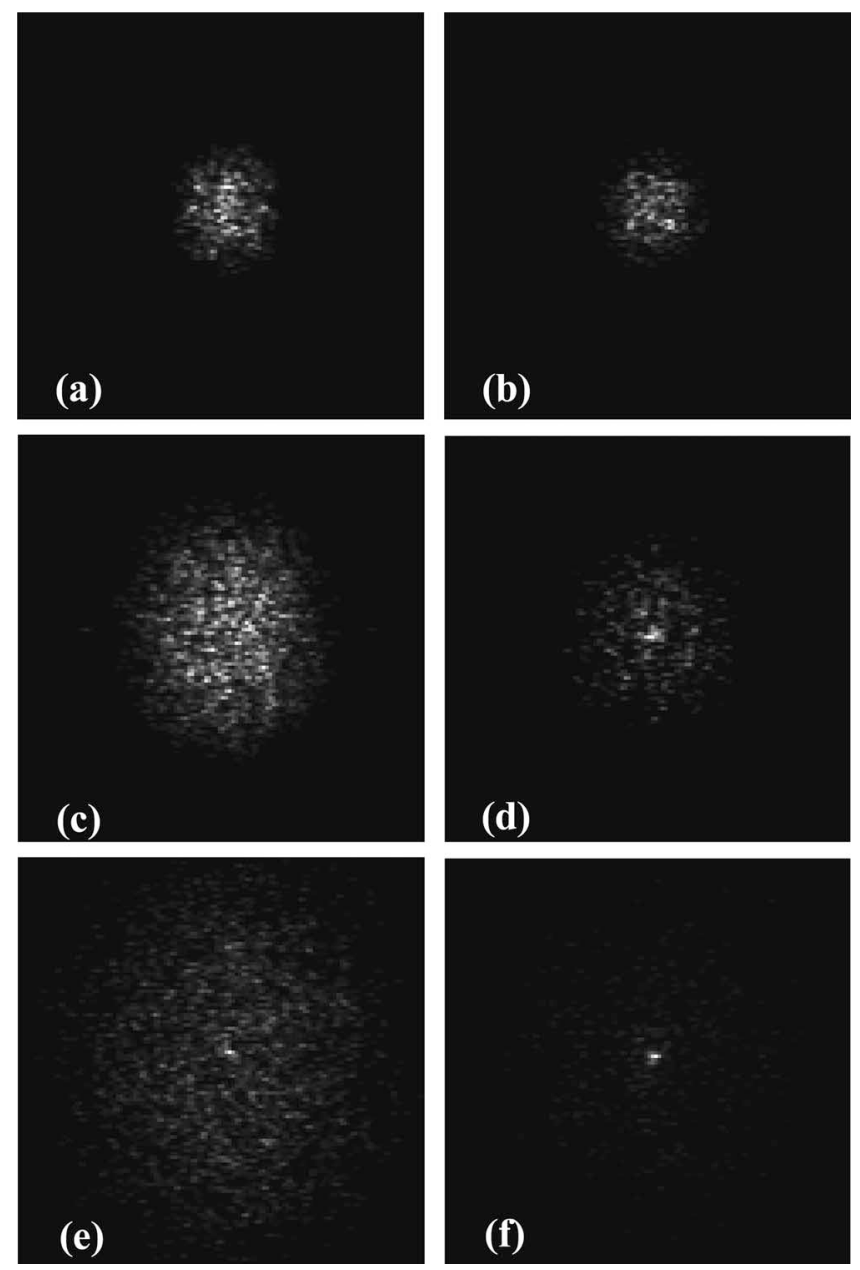

Fig. 2. FT obtained from randomized binary data pages: (a), (c), and (e), using $\pi$ BPM (gray levels 0 and 198); (b), (d), and (f), using HTM (gray levels 0, 193, and 250). The bit size is $16 \times 16$ pixels in (a) and (b), $8 \times 8$ pixels in (c) and (d), and $4 \times 4$ pixels in (e) and (f).

and 198 in the case of $\pi \mathrm{BPM}$, and 0,193 , and 250 in the case of the HTM. In Fig. 2 we show the experimental images obtained for the FT of the randomized binary data pages. The two columns correspond, respectively, to the results obtained with $\pi \mathrm{BPM}$ and HTM. The bits in the randomized data pages have been produced with different sizes. In the first, second, and third rows we show, respectively, the results for a bit size of $16 \times 16,8 \times 8$, and $4 \times 4$ LCD pixels. The intensity of the laser beam has been controlled so as not to saturate the CCD camera. Then, the central region of the original images has been extracted and the histogram has been scaled to cover the graylevel range from 0 to 255 . In the case of the $\pi \mathrm{BPM}$ we see that the FT is very homogeneous for the three bit sizes, even though a weak dc peak becomes visible in the case of the $4 \times 4$ bit size [Fig. 2(e)]. In the case of HTM a weak dc peak appears in the case of the 8 $\times 8$ bit size [Fig. 2(d)], and becomes stronger in the case of the $4 \times 4$ bit size [Fig. 2(f)], even though it is still much lower than the dc peak produced by a binary amplitude data page. The appearance of the dc peaks at smaller bit sizes is partly due to the anamorphic frequency dependent effect already commented upon: the optimum gray levels producing a $\pi$ radians phase difference are different along the vertical and horizontal direction, and this gray-level difference gets larger with the increase in the spatial frequency components of the image addressed onto the LCD [10]. This effect is stronger for HTM since the amplitude transmission is not constant along the whole gray-level range as in the case of $\pi \mathrm{BPM}$. Therefore, the anamorphic frequency dependent effect produces both a deviation of amplitude transmission and phase-shift values.

In conclusion, we have demonstrated an accurate procedure to eliminate the dc peak in the FT of binary data pages in HDSS using a commercial LCD. The accuracy of the model used for the LCD allows a reliable computer search to obtain $\pi \mathrm{BPM}$ or HTM configurations. Some final adjustments in the voltage levels may be necessary, which can be easily performed using binary phase gratings written onto the LCD.

We acknowledge financial support from the Spanish Ministerio de Educación y Ciencia (grants FIS2005-05881-C02-01 and FIS2005-05881-C02-02), and from Generalitat Valenciana (grant GV06-007).

\section{References}

1. P. Yeh and C. Gu, Optics of Liquid Crystal Displays (Wiley, 1999).

2. H. J. Coufal, D. Psaltis, and B. T. Sincerbox, eds., Holographic Data Storage (Springer-Verlag, 2000).

3. R. K. Kostuk, M. P. Bernal, and Q. Cao, in Holographic Data Storage, H. J. Coufal, D. Psaltis, and G. T. Sincerbox, eds. (Springer-Verlag, 2000).

4. J.-S. Jang and D.-H. Shin, Opt. Lett. 26, 1797 (2001).

5. J. Reméyi, P. Várhegyi, L. Domján, P. Koppa, and E. Lorincz, Appl. Opt. 42, 3428 (2003).

6. R. John, J. Joseph, and K. Singh, Opt. Lasers Eng. 43, 183 (2005).

7. J. Joseph and D. A. Waldman, Appl. Opt. 45, 6374 (2006)

8. A. Márquez, C. Iemmi, I. Moreno, J. A. Davis, J. Campos, and M. J. Yzuel, Opt. Eng. 40, 2558 (2001).

9. A. Márquez, J. Campos, M. J. Yzuel, I. Moreno, J. A. Davis, C. Iemmi, A. Moreno, and A. Robert, Opt. Eng. 39, 3301 (2000).

10. A. Márquez, I. Moreno, C. Iemmi, J. Campos, and M. J. Yzuel, Opt. Eng. (to be published). 